PROCEEDINGS OF THE

AMERICAN MATHEMATICAL SOCIETY

Volume 136, Number 6, June 2008, Pages 2183-219

S 0002-9939(08)09225-3

Article electronically published on February 19, 2008

\title{
COHOMOLOGY \\ IN ONE-DIMENSIONAL SUBSTITUTION TILING SPACES
}

\author{
MARCY BARGE AND BEVERLY DIAMOND
}

(Communicated by Jane M. Hawkins)

\begin{abstract}
Anderson and Putnam showed that the cohomology of a substitution tiling space may be computed by collaring tiles to obtain a substitution which "forces its border." One can then represent the tiling space as an inverse limit of an inflation and substitution map on a cellular complex formed from the collared tiles; the cohomology of the tiling space is computed as the direct limit of the homomorphism induced by inflation and substitution on the cohomology of the complex. For one-dimensional substitution tiling spaces, we describe a modification of the Anderson-Putnam complex on collared tiles that allows for easier computation and provides a means of identifying certain special features of the tiling space with particular elements of the cohomology.
\end{abstract}

\section{INTRODUCTION}

The investigation of the topology of substitution tiling spaces, in particular, the study of the cohomology of the space, is often aided by representing the tiling space as the inverse limit of an inflation and substitution map on the cellular AndersonPutnam complex $([\mathrm{AP}])$. If the substitution forces its border, the complex can be formed using the given tiles as cells, and adding identifications indicating allowed transitions; the cohomology of the tiling space is computed as the direct limit of the homeomorphism induced by inflation and substitution on the cohomology of the complex. If the substitution does not force its border, then the inverse limit constructed in this way may be a nontrivial quotient of the tiling space rather than the tiling space itself; certain arc components may be glued together, and others pinched at points, in some cases changing the cohomology. In this case, the process of collaring provides a larger set of tiles and an induced substitution which does force its border. The inverse limit space of the complex formed from the larger set of tiles and associated map is homeomorphic to the tiling space of the original substitution.

In many situations, full collaring leads to a very large number of tiles, and the process of determining the complex can be tedious and time-consuming. In addition, the cohomology of the tiling space is computed as the direct limit of the transpose of a single (large) transition matrix, rather than in pieces that have some topological interpretation. We indicate below, for one-dimensional substitution tilings (that is, the tiles are subintervals of $\mathbb{R}$ ), a process which achieves the same

Received by the editors February 14, 2007, and, in revised form, May 4, 2007.

2000 Mathematics Subject Classification. Primary 37B05; Secondary 37A30, 37B50, 54H20.

(C) 2008 American Mathematical Society

Reverts to public domain 28 years from publication 
ultimate goal, that of representing the tiling space as an inverse limit on an associated, simple complex, from which the cohomology can be computed in a fairly straightforward way; the approach we present yields a specific, concrete, topological interpretation of a piece of the cohomology and the relationship between that contribution and the remaining cohomology (see Theorems 4 and $4^{\prime}$ ).

We made use of rewriting a one-dimensional substitution in [BD], based on ideas introduced in Dur, to modify an arbitrary one-dimensional substitution to obtain a proper substitution that then automatically forces its border and has a tiling space homeomorphic to that of the original substitution. The modified proper substitution typically has a smaller alphabet than that of the substitution produced by collaring, hence the cohomology of the original tiling space may be more efficiently computed by rewriting than by collaring. This process still has two drawbacks in our view. First, there is no analog of rewriting in higher dimensions, while the construction we describe below generalizes easily to higher dimensions. Second, with rewriting, as with collaring, the cohomology is calculated as the direct limit of a single matrix, disguising the contribution of certain topological features.

\section{Notation AND TERMinOlOGY}

We introduce some notation and terminology.

Let $\mathcal{A}=\{1,2, \ldots, \operatorname{card}(\mathcal{A})\}$ be a finite alphabet; $\mathcal{A}^{*}$ will denote the collection of finite nonempty words with letters in $\mathcal{A}$. A substitution on $\mathcal{A}$ is a map $\varphi: \mathcal{A} \rightarrow \mathcal{A}^{*}$; $\varphi$ extends naturally to $\varphi: \mathcal{A}^{*} \rightarrow \mathcal{A}^{*}$. The transition matrix $A_{\varphi}=A$ for $\varphi$ is $A:=\left(a_{i j}\right)_{i \in \mathcal{A}, j \in \mathcal{A}}$ in which $a_{i j}$ is the number of occurrences of $i$ in the word $\varphi(j)$.

The substitution $\varphi$ is primitive if $\varphi^{n}(i)$ contains $j$ for all $i, j \in \mathcal{A}$ and sufficiently large $n$. Equivalently, $\varphi$ is primitive if and only if the matrix $A$ is aperiodic, in which case $A$ has a simple eigenvalue $\lambda_{\varphi}$ larger in modulus than its remaining eigenvalues called the Perron-Frobenius eigenvalue of $A$ (and $\varphi$ ).

A word $w$ is allowed for $\varphi$ if and only if for each finite subword (i.e., factor) $w^{\prime}$ of $w$, there are $i \in \mathcal{A}$ and $n \in \mathbb{N}$ such that $w^{\prime}$ is a subword of $\varphi^{n}(i)$; the language of $\varphi, \mathcal{L}_{\varphi}=\mathcal{L}$, is the set of finite allowed words for $\varphi$. Let $W_{\varphi}$ denote the set of allowed bi-infinite words for $\varphi$. We identify the $0^{\text {th }}$ coordinate in a bi-infinite word $w$ by either an indexing, as in $w=\ldots w_{-1} w_{0} w_{1} \ldots$, or by use of a decimal point (or both). Let $\sigma: W_{\varphi} \rightarrow W_{\varphi}$ denote the shift map:

$$
\sigma\left(\ldots w_{-1} \cdot w_{0} w_{1} \ldots\right):=\ldots w_{-1} w_{0} \cdot w_{1} \ldots
$$

The substitution $\varphi: \mathcal{A} \rightarrow \mathcal{A}^{*}$ extends to $\varphi: W_{\varphi} \rightarrow W_{\varphi}$ where

$$
\varphi\left(\ldots w_{-1} w_{0} w_{1} \ldots\right):=\ldots \varphi\left(w_{-1}\right) \cdot \varphi\left(w_{0}\right) \varphi\left(w_{1}\right) \ldots
$$

The word $w$ is periodic for $\varphi$ under inflation and substitution, or $\varphi$-periodic, if for some $m \in \mathbb{N}$,

$$
\varphi^{m}(w)=\ldots \varphi^{m}\left(w_{-1}\right) \cdot \varphi^{m}\left(w_{0}\right) \varphi^{m}\left(w_{1}\right) \ldots=\ldots w_{-1} . w_{0} w_{1} \ldots
$$

Each primitive substitution $\varphi$ has at least one allowed $\varphi$-periodic bi-infinite word which is necessarily uniformly recurrent under the shift. A substitution $\varphi$ with precisely one periodic, hence fixed, bi-infinite word is called proper; $\varphi$ is proper if and only if there are $b, e \in \mathcal{A}$ such that for all sufficiently large $k$ and all $i \in \mathcal{A}$, $\varphi^{k}(i)=b \ldots e$.

A primitive substitution $\varphi$ is aperiodic if at least one (equivalently, each) $\varphi$ periodic bi-infinite word is not periodic under the natural shift map, in which case 
$\left(W_{\varphi}, \sigma\right)$ is an infinite minimal dynamical system. If $\varphi$ is aperiodic, then the map $\varphi: W_{\varphi} \rightarrow W_{\varphi}$ is one-to-one ([Mo]). If $\varphi$ is periodic (that is, primitive and not aperiodic), then $W_{\varphi}$ is finite.

Given a primitive substitution $\varphi: \mathcal{A} \rightarrow \mathcal{A}^{*}$ with $\operatorname{card}(\mathcal{A})=d \geq 2$, let $\omega_{L}:=$ $\left(\omega_{1}, \ldots, \omega_{d}\right)$ be a positive left eigenvector for the Perron-Frobenius eigenvalue, $\lambda$, of $A$. The intervals $P_{i}=\left[0, \omega_{i}\right], i=1, \ldots, d$, are called prototiles for $\varphi$ (consider $P_{i}$ to be distinct from $P_{j}$ for $i \neq j$ even if $\omega_{i}=\omega_{j}$ ). A tiling $T$ of $\mathbb{R}$ by the prototiles for $\varphi$ is a collection $T=\left\{T_{i}\right\}_{i=-\infty}^{\infty}$ of tiles $T_{i}$ for which $\bigcup_{i=-\infty}^{\infty} T_{i}=\mathbb{R}$, each $T_{i}$ is a translate of some $P_{j}$ (in which case we say $T_{i}$ is of type $j$ ), and $T_{i} \cap T_{i+1}$ is a singleton for each $i$. Generally we assume that the indexing is such that $0 \in T_{0} \backslash T_{1}$.

If $\varphi(i)=i_{1} i_{2} \ldots i_{k(i)}$, then $\lambda \omega_{i}=\sum_{j=1}^{k(i)} \omega_{i_{j}}$. Thus $\left|\lambda P_{i}\right|=\sum_{j=1}^{k(i)}\left|P_{i_{j}}\right|$, and $\lambda P_{i}$ is tiled by $\left\{T_{j}\right\}_{j=1}^{k(i)}$, where $T_{j}=P_{i_{j}}+\sum_{k=1}^{j-1} \omega_{i_{k}}$. This process is called inflation and substitution and extends to a map $\Phi$ taking a tiling $T=\left\{T_{i}\right\}_{i=-\infty}^{\infty}$ of $\mathbb{R}$ by prototiles to a new tiling, $\Phi(T)$, of $\mathbb{R}$ by prototiles defined by inflating, substituting, and suitably translating each $T_{i}$. More precisely, for $w=w_{1} \ldots w_{n} \in \mathcal{A}^{*}$, define

$$
\mathcal{P}_{w}+t=\left\{P_{w_{1}}+t, P_{w_{2}}+t+\left|P_{w_{1}}\right|, \ldots, P_{w_{n}}+t+\Sigma_{i<n}\left|P_{w_{i}}\right|\right\} .
$$

Then $\Phi\left(P_{i}+t\right)=\mathcal{P}_{\varphi(i)}+\lambda t$ and $\Phi\left(\left\{P_{k_{i}}+t_{i}\right\}_{i \in \mathbb{Z}}\right)=\bigcup_{i \in \mathbb{Z}}\left(\mathcal{P}_{\varphi\left(k_{i}\right)}+\lambda t_{i}\right)$.

There is a natural topology on the collection $\Sigma_{\varphi}$ of all tilings of $\mathbb{R}$ by prototiles $\left(\left\{T_{i}\right\}_{i=-\infty}^{\infty}\right.$ and $\left\{T_{i}^{\prime}\right\}_{i=-\infty}^{\infty}$ are "close" if there is an $\epsilon$ near 0 so that $\left\{T_{i}\right\}_{i=-\infty}^{\infty}$ and $\left\{T_{i}^{\prime}+\epsilon\right\}_{i=-\infty}^{\infty}$ are identical in a large neighborhood of 0 (see AP for details)). The space $\Sigma_{\varphi}$ is compact and metrizable with this topology and $\Phi: \Sigma_{\varphi} \rightarrow \Sigma_{\varphi}$ is continuous. Given $T=\left\{T_{i}\right\}_{i=-\infty}^{\infty} \in \Sigma_{\varphi}$, let $\underline{w}(T)=\ldots w_{-1} w_{0} w_{1} \ldots$ denote the bi-infinite word with $w_{i}=j$ if and only if $T_{i}$ is of type $j$. The tiling space associated with $\varphi, \mathcal{T}_{\varphi}$, is defined as

$$
\mathcal{T}_{\varphi}=\{T: \underline{w}(T) \text { is allowed for } \varphi\} .
$$

There is a natural flow (translation) on $\Sigma_{\varphi}$ defined by $\left(\left\{T_{i}\right\}_{i=-\infty}^{\infty}, t\right) \mapsto$ $\left\{T_{i}-t\right\}_{i=-\infty}^{\infty}$. If $\varphi$ is primitive and aperiodic, $\Phi: \mathcal{T}_{\varphi} \rightarrow \mathcal{T}_{\varphi}$ is a homeomorphism (this relies on the notion of recognizability or invertibilty for such substitutions; see [Mo] and $\left[\mathrm{So}\right.$ ). Each $T \in \mathcal{T}_{\varphi}$ is uniformly recurrent under the flow and has dense orbit (i.e., the flow is minimal on $\mathcal{T}_{\varphi}$ ). It follows that $\mathcal{T}_{\varphi}$ is a continuum.

Recall that a composant of a point $x$ in a topological space $X$ is the union of the proper compact connected subsets of $X$ containing $x$. If $\varphi$ is a primitive substitution, composants and arc components in $\mathcal{T}_{\varphi}$ are identical; in this case we use the terms interchangeably. For any substitution $\varphi$, the arc components of the tiling space $\mathcal{T}_{\varphi}$ coincide with the orbits of the natural flow (translation) on $\mathcal{T}_{\varphi}$.

Tilings $T, T^{\prime} \in \mathcal{T}_{\varphi}$ are forward asymptotic if $\lim _{t \rightarrow \infty} \operatorname{dist}\left(T-t, T^{\prime}-t\right)=0$. Equivalently, $T=\left\{T_{i}\right\}_{i=-\infty}^{\infty}, T^{\prime}=\left\{T_{i}^{\prime}\right\}_{i=-\infty}^{\infty}$ are forward asymptotic if there are $N, M \in \mathbb{Z}$ so that $T_{N+k}=T_{M+k}^{\prime}$ for all $k \geq 0$. Composants are forward asymptotic if they contain forward asymptotic tilings. Backward asymptotic tilings and composants are defined similarly.

If $f: X \rightarrow X$ is a map of a compact connected metric space $X$, then the inverse limit space with single bonding map $f$ is the space

$$
\varliminf_{\longleftarrow} f=\left\{\left(x_{0}, x_{1}, \ldots\right): f\left(x_{i}\right)=x_{i-1} \text { for } i=1,2, \ldots\right\}
$$

with metric

$$
\underline{d}(\underline{x}, \underline{y})=\sum_{i \geq 0} \frac{d\left(x_{i}, y_{i}\right)}{2^{i}}
$$


$\hat{f}: \lim _{f} f \rightarrow \lim f$ will denote the natural (shift) homeomorphism

$$
\hat{f}\left(x_{0}, x_{1}, \ldots\right)=\left(f\left(x_{0}\right), x_{0}, x_{1}, \ldots\right) .
$$

\section{The MOdified COMPLEX AND ITS COHOMOLOGY}

Given a primitive, aperiodic substitution $\varphi$ on $d$ letters, the complex $K=K_{\varphi}$ will consist of a collection of edges representing the letters of $\mathcal{A}$, another collection of edges representing allowed transitions between letters, and certain identifications.

As above, $\omega_{L}=\left(\omega_{1}, \ldots, \omega_{d}\right)$ is a left eigenvector for the Perron-Frobenius eigenvalue $\lambda$ for $\varphi$. Define $0<\epsilon=\min \left\{\frac{\omega_{a}}{2 \lambda}\right\}_{a \in \mathcal{A}}$. For $a \in \mathcal{A}$, let

$$
e_{a}:=\left[\epsilon, \omega_{a}-\epsilon\right] \times\{a\},
$$

and for $a b \in \mathcal{L}$, let

$$
e_{a b}:=[-\epsilon, \epsilon] \times\{a b\} .
$$

Define

$$
K:=\left[\left(\bigcup_{a \in \mathcal{A}} e_{a}\right) \cup\left(\bigcup_{a b \in \mathcal{L}} e_{a b}\right)\right] / \sim
$$

where

$$
\left(\omega_{a}-\epsilon, a\right) \sim(-\epsilon, a b)
$$

and

for all $a, b \in \mathcal{A}$, and let

$$
(\epsilon, b) \sim(\epsilon, a b)
$$

$$
S:=\bigcup_{a b \in \mathcal{L}} e_{a b} / \sim
$$

Loosely, the complex $K$ is a wedge of $d$ circles with the branch point "blown up" to $S ; K / S$ is homeomorphic to the wedge of $d$ circles.

Example 1. The Fibonacci substitution: If $\varphi$ is the Fibonacci substitution $(\varphi(1)=$ $12, \varphi(2)=1$ ), then all transitions except 22 are allowed, and $K_{\varphi}$ is (topologically and not to scale) shown below.

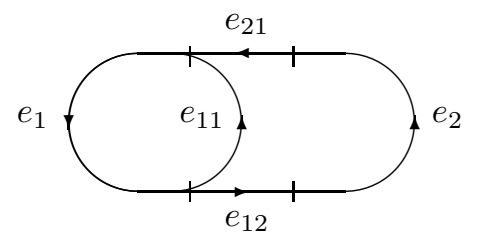

In Example1, collaring leads to a topologically identical complex. In the general case, the complex $K$ constructed above is a quotient of the complex on collared tiles, usually nontrivial.

Given a tiling $T=\left\{T_{n}\right\}_{n \in \mathbb{Z}} \in \mathcal{T}_{\varphi}$, let $t \in \mathbb{R}$ and $a, b, c \in \mathcal{A}$ be such that $0 \in T_{0}=\left[0, \omega_{a}\right]-t, T_{-1}=\left[0, \omega_{b}\right]-t-\omega_{b}$, and $T_{1}=\left[0, \omega_{c}\right]-t+\omega_{a}$. Define a map $p: \mathcal{T}_{\varphi} \rightarrow K$ as follows:

$$
p(T)= \begin{cases}{[(t, a)]} & \text { if } \epsilon \leq t \leq \omega_{a}-\epsilon \\ {[(t, b a)]} & \text { if } 0 \leq t \leq \epsilon \\ {\left[\left(t-\omega_{a}, a c\right)\right]} & \text { if } \omega_{a}-\epsilon \leq t \leq \omega_{a}\end{cases}
$$

The map $p$ is a continuous surjection, and there is a unique map $f: K \rightarrow K$ such that $f \circ p=p \circ \Phi$, where $\Phi: \mathcal{T}_{\varphi} \rightarrow \mathcal{T}_{\varphi}$ is the inflation and substitution 
homeomorphism. It is easy to verify that if $\varphi(a)=a_{1} a_{2} \ldots a_{k}$, then $f\left(e_{a}\right) \subset$ $e_{a_{1}} \cup e_{a_{1} a_{2}} \cup e_{a_{2}} \cup \ldots \cup e_{a_{k-1}} \cup e_{a_{k-1} a_{k}} \cup e_{a_{k}}$, and if $\varphi(a . b)=\ldots c . d \ldots$, then $f\left(e_{a b}\right) \subseteq e_{c} \cup e_{c d} \cup e_{d}$.

Lemma 2. $\lim _{\longleftarrow} f \simeq \mathcal{T}_{\varphi}$.

Proof: The commuting diagram

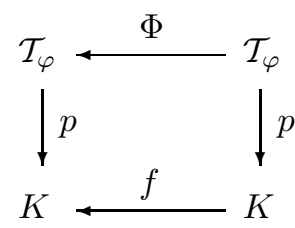

induces a map $\hat{p}: \mathcal{T}_{\varphi} \rightarrow \lim f$ given by $\hat{p}(T)=\left(p(T), p\left(\Phi^{-1}(T)\right), \ldots\right)$.

Let $V:=\{(\epsilon, a)\}_{a \in \mathcal{A}} \overleftarrow{\cup}\left\{\left(\omega_{a}-\epsilon, a\right)\right\}_{a \in \mathcal{A}}$, and choose $\underline{x}=\left(x_{0}, x_{1}, \ldots\right) \in \lim f$. Suppose that for some $i \in \mathbb{N}, x_{i} \in V$. The choice of $\epsilon$ implies that $x_{i-1} \in e_{b} \backslash \overleftarrow{V}$ for some $b \in \mathcal{A}$. That is, if $T \in \hat{p}^{-1}(\underline{x})$, then the $0^{t h}$ tile of $\Phi^{1-i}(T)$, and the position of the origin within the interior of this tile, are determined, as are segments of $T$ of approximate length $\lambda^{i} \omega_{b}$ about the origin. It follows that $T \in \hat{p}^{-1}(\underline{x})$ is totally determined if $x_{i} \in V$ for arbitrarily large $i$.

If $x_{i} \notin V$ for arbitrarily large $i$, then for sufficiently large $n \in \mathbb{N}$, either $x_{n} \in e_{a b}$ for some $a, b \in \mathcal{A}$ or $x_{n} \in e_{b} \backslash V$ for some $b \in \mathcal{A}$. In the first case, the $(-1)^{s t}$ and $0^{t h}$ tiles of $\Phi^{1-n}(T)$, and the position of the origin within the interior of the union of these edges, are determined. In both cases, arguments identical to those above imply that arbitrarily large segments of $T$ about the origin are determined, thus $T$ is totally determined, and $\hat{p}$ is one-to-one. As $p$ is onto, so is $\hat{p}$.

There are two essential ways in which the cohomology of the inverse limit of the natural map on the wedge of circles, that is, $\lim _{\varphi} A_{\varphi}^{t}$, may need to be modified to obtain the cohomology of the tiling space $\mathcal{T}_{\varphi}$.

First, if $\mathcal{T}_{\varphi}$ contains one or more cycles of asymptotic composants associated with a cycle of $\varphi$-periodic words of the form $\ldots a_{1} . a_{2} \ldots, \ldots a_{3} . a_{2} \ldots, \ldots a_{3} . a_{4} \ldots$, $\ldots, \ldots a_{1} \cdot a_{2 n} \ldots$, then those composants are "glued together" in the inverse limit on the wedge of circles to form $n$-ods (possibly overlapping). The modified collaring described above, in particular, the construction of the subcomplex $S$, "pulls apart" these cycles. The cohomology associated with the unglued cycles in $\mathcal{T}_{\varphi}$ is computed via $\check{H}^{1}(S)$ and must be added to $\lim _{\longrightarrow} A^{t}$.

Second, there may be "extra" cohomology appearing in $\lim _{\longrightarrow} A^{t}$ that does not appear in $\mathcal{T}_{\varphi}$ that we identify below.

We find it easier to compute the cohomology of $\mathcal{T}_{\varphi}$ by working with a map $g$ defined on $K$ that is homotopic to $f$ and leaves the transition edges invariant. We define $g$ as follows.

First, let $\delta=\min \left\{\frac{\omega_{a}}{3}\right\}_{a \in \mathcal{A}}$. For $a \in \mathcal{A}$, define

$$
h_{a}(x)= \begin{cases}\epsilon & \text { if } \epsilon \leq x \leq \delta \\ \epsilon+\frac{\omega_{a}-2 \epsilon}{\omega_{a}-2 \delta}(x-\delta) & \text { if } \delta \leq x \leq \omega_{a}-\delta \\ \omega_{a}-\epsilon & \text { if } \omega_{a}-\delta \leq x \leq \omega_{a}-\epsilon .\end{cases}
$$

That is, $h_{a}$ linearly maps the interval $\left[\delta, \omega_{a}-\delta\right]$ over $\left[\epsilon, \omega_{a}-\epsilon\right]$ and collapses the intervals $[\epsilon, \delta]$ and $\left[\omega_{a}-\delta, \omega_{a}-\epsilon\right]$ to $\{\epsilon\}$ and $\left\{\lambda_{a}-\epsilon\right\}$, respectively. 
Let $h: K \rightarrow K$ be defined as:

$$
h(x, w)= \begin{cases}\left(h_{a}(x), w\right) & \text { if } x \in e_{a}, \text { for } a \in \mathcal{A}, \\ (x, w) & \text { if } x \in e_{a b}, \text { for } a b \in \mathcal{L} .\end{cases}
$$

Finally, $g:=h \circ f$. If $\varphi(a . b)=\ldots$ c.d $\ldots$, then $g\left(e_{a b}\right)=e_{c d}$, hence $g(S) \subseteq S$.

We show below that the "extra" piece of cohomology mentioned above may be computed as follows. We find the eventual range $E R$ of $\left.g\right|_{S}$, and the rank of the group generated by the coboundaries of the components of $E R$. Each generator for this group is an eigenvector associated with eigenvalue 1 for a power of $g^{*}$ acting on $H^{1}(K, S)$, and the rank of the group indicates the number of copies of $\mathbb{Z}$ to be quotiented out from $\lim A^{t}$.

Whereas $\lim f \simeq \overrightarrow{\mathcal{T}_{\varphi}}, \underline{\lim } g$ is homeomorphic to a quotient of $\mathcal{T}_{\varphi}$ in which asymptotic composants for $\varphi$ associated with $\varphi$-periodic words, should there be any, are glued in the direction of asymptoticity (each such pair of composants is associated with a pair of $\varphi$-periodic words of the form ...a.b..., ..a.c..., thus with the edges $e_{a}, e_{a b}$ and $e_{a c}$, with the last two periodic under $g$ ). However, in this case, unlike the situation with the inverse limit space on the wedge of circles, gluing does not extend all the way to the origins of the associated tilings, and the next result says that this gluing does not change the cohomology of $\mathcal{T}_{\varphi}$.

Corollary 3. $\underline{\lim }_{\longrightarrow} g^{*} \simeq \check{H}\left(\mathcal{T}_{\varphi}\right)$.

Proof. Since $f$ and $g$ are homotopic, $\lim _{\longrightarrow} g^{*}=\lim _{\longrightarrow} f^{*} \simeq \check{H}\left(\mathcal{T}_{\varphi}\right)$.

Denote the eventual range $\bigcap_{n>0} g^{n}(S)$ of $\left.g\right|_{S}$ by $E R$. Let $k$ be the number of connected components of $E R$ and $l$ the number of independent 1-cycles in $E R$; we call $l$ the asymptotic cycle rank of $\varphi$. (Note that if $\varphi$ is proper, $E R$ consists of a single edge $e_{a b}$. If $\varphi$ forces its border, then each component of $E R$ is a single edge.)

Our main result is the following:

Theorem 4. $\check{H}^{1}\left(\mathcal{T}_{\varphi}\right) \simeq \mathbb{Z}^{l} \oplus \underline{\lim } A^{t} / G$, where $G \simeq \mathbb{Z}^{k-1}$.

Proof. There is an $m \in \mathbb{N}$ such that $g^{m}(C)=C$ for each component $C$ of $E R$ and $g^{m}(S) \subseteq E R$. Pick a point $c$ from each component $C$ of $S$, and let $\hat{c}$ denote the corresponding dual generator in $H^{0}(S)$. For each component $C$ of $E R$, the element

$$
v_{C}=\sum\left\{\hat{c}^{\prime}: C^{\prime} \text { is a component of } S, g^{m}\left(C^{\prime}\right) \subseteq C\right\}
$$

is fixed by $\left(g^{*}\right)^{m}: H^{0}(S) \rightarrow H^{0}(S)$. Furthermore, the eventual range of $g^{*}$ has basis $\left\{v_{C}: C\right.$ a component of $\left.E R\right\}$, and the sum of these basis elements, $\sum v_{C}$, generates the range $\eta$ of the augmentation map. Thus, choosing $k-1$ of the components, $C_{1}, \ldots, C_{k-1}$, of $E R$, the cosets $\left\{v_{C_{1}}+\eta, \ldots, v_{C_{k-1}}+\eta\right\}$ form a basis for the eventual range of $g^{*}$ on the reduced cohomology $\tilde{H}^{0}(S):=H^{0}(S) / \eta$, and each of these basis elements is an eigenvector for $\left(g^{*}\right)^{m}$ with eigenvalue 1.

It is clear that $g^{m}$ is homotopic to the identity on $E R$, so that $\lim _{\longrightarrow} g^{*}: H^{1}(S) \rightarrow$ $H^{1}(S) \simeq \mathbb{Z}^{l}$, where $l$ is the asymptotic cycle rank of $\varphi$. To see how the 1 -cycles of $E R$ contribute to the cohomology of $\mathcal{T}_{\varphi}$, consider the morphism of the exact 
sequence of the pair $(K, S)$ induced by $g:(K, S) \rightarrow(K, S)$ :

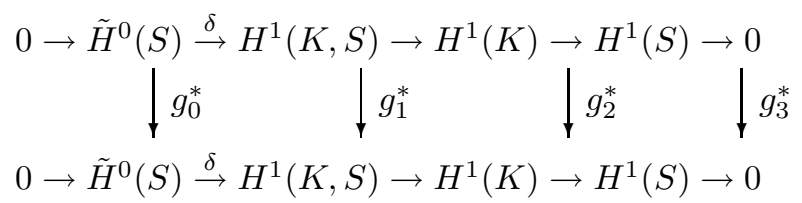

Taking direct limits, we get an exact sequence

$$
0 \rightarrow G_{0} \stackrel{\vec{\delta}}{\rightarrow} G_{1} \rightarrow G_{2} \rightarrow G_{3} \rightarrow 0
$$

in which $G_{1} \simeq \lim _{\longrightarrow} A^{t}, G_{2}:=\lim _{\longrightarrow} g_{2}^{*} \simeq \check{H}^{1}\left(\mathcal{T}_{\varphi}\right), G_{3}:=\lim _{\longrightarrow} g_{3}^{*} \simeq \mathbb{Z}^{l}$, and $G_{0}:=$

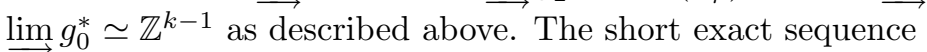

$$
0 \rightarrow \operatorname{coker}(\vec{\delta}) \rightarrow G_{2} \rightarrow G_{3} \rightarrow 0
$$

splits to give $\check{H}^{1}\left(\mathcal{T}_{\varphi}\right) \simeq \operatorname{coker}(\vec{\delta}) \oplus \mathbb{Z}^{l}$.

Since $H^{1}(K)$ is free, so is coker $(\delta)$. In particular, the image under $\delta$ of a basis of $\tilde{H}^{0}(S)$,

$$
\left\{\delta\left(\hat{c}_{1}+\eta\right), \ldots, \delta\left(\hat{c}_{k-1}+\eta\right)\right\} \cup\{\delta(\hat{c}+\eta): C \text { a component of } S, C \cap E R=\emptyset\},
$$

extends to a basis for $H^{1}(K, S)$ with respect to which $\left(g_{1}^{*}\right)^{m}$ takes the form

$$
\left(\begin{array}{ccc}
I & 0 & E_{2} \\
E_{1} & 0 & E_{3} \\
0 & 0 & A_{1}
\end{array}\right),
$$

where $I$ is the $(k-1) \times(k-1)$ identity matrix, and the diagonal 0 matrix is size $j \times j$, where $j:=\left(\#\right.$ of components in $S$ ) $-k$. Note that in the basis $\left\{\hat{e}_{a}\right.$ : $a \in \mathcal{A}\}, g_{1}^{*}$ is represented by $A^{t}$, the transpose of the transition matrix for $\varphi$. Thus $(*)$ is unimodularly equivalent to $\left(A_{\varphi}^{t}\right)^{m}$, and $\operatorname{coker}(\vec{\delta}) \simeq \underset{\lim }{\longrightarrow}\left(\overline{g_{1}^{*}}\right)^{m}$, where

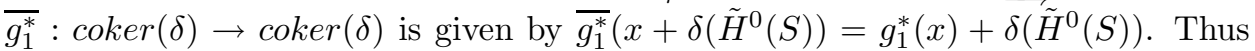
$\operatorname{coker}(\vec{\delta}) \simeq \lim _{\longrightarrow} A_{1}$ and we have $\check{H}^{1}\left(\mathcal{T}_{\varphi}\right) \simeq \underline{\lim } A_{1} \oplus \mathbb{Z}^{l}$.

Note: $\underline{\lim }\left(\begin{array}{cc}I & 0 \\ E_{1} & 0\end{array}\right) \simeq \mathbb{Z}^{k-1}$. There are conditions that imply that $\underline{\longrightarrow} A^{t}$ splits as $\mathbb{Z}^{k-1} \oplus \underline{\lim } A_{1}$. For instance, if there is a matrix $B$ so that

$$
B A_{1}+\left(\begin{array}{cc}
I & 0 \\
E_{1} & 0
\end{array}\right)=-\left(\begin{array}{c}
E_{1} \\
E_{2}
\end{array}\right)
$$

then $\lim A^{t}$ splits as described. We do not know if there is always such a splitting.

We rephrase the above discussion in a way that is more consistent with actual computations (for instance, see Example 8) and allows for a more precise statement of Theorem 4 ,

Let $f_{i}=(0 \ldots 010 \ldots)^{t}$ be the $i^{t h}$ standard basis vector for $\mathbb{R}^{d}$ and $C_{1}, \ldots, C_{p}$ the components of the complex $S$. For $i=1, \ldots, p-1$, let $w_{i}:=\sum f_{j}-\sum f_{m}$, where the first sum is over those $j$ for which $e_{j}=\left[\epsilon, \lambda_{j}-\epsilon\right] \times\{j\}$ terminates in $C_{i}$ (i.e., $\left(\lambda_{j}-\right.$ $\epsilon, j) \in C_{i}$ ) and the second sum is over those $m$ for which $e_{m}$ originates in $C_{i}$ (i.e., $\left.(\epsilon, m) \in C_{i}\right)$. Then $\left\{w_{1}, \ldots, w_{p-1}\right\}$ extends to a basis $\left\{w_{1}, \ldots, w_{p-1}, w_{p}, \ldots, w_{d}\right\}$ of $\mathbb{Z}^{d}$. Let $P$ be the matrix with columns $\left\{w_{1}, \ldots, w_{p-1}, w_{p}, \ldots, w_{d}\right\}$. Then $P^{-1} A^{t} P$ has the form $\left(\begin{array}{cc}E & F \\ 0 & A_{1}\end{array}\right)$ in which $A_{1}$ is size $(d-p+1) \times(d-p+1)$. 
Theorem $4^{\prime}$. Let $\varphi$ be an aperiodic primitive substitution with asymptotic cycle rank $l$ and $A_{1}$ as above. Then $\check{H}^{1}\left(\mathcal{T}_{\varphi}\right) \simeq \lim _{\longrightarrow} A_{1} \oplus \mathbb{Z}^{l}$.

Corollary 5. Let $\varphi, K$, and $S$ be as above. If $\tilde{H}^{*}(S) \simeq 0$, then $\check{H}^{1}\left(\mathcal{T}_{\varphi}\right) \simeq \underline{\lim } A_{\varphi}^{t}$.

Example 6. The Fibonacci substitution: If $\varphi$ is the Fibonacci substitution $(\varphi(1)=$ $12, \varphi(2)=1$ ) (see Example 1), then $S$ has a single connected component, and the eventual range of $\left.g\right|_{S}$ is the connected pair of edges $E R=e_{11} \cup e_{21}$, thus $l=0$, $k=1$. By Theorem 4 .

$$
\check{H}^{1}\left(\mathcal{T}_{\varphi}\right) \simeq \lim _{\longrightarrow}\left[\left(\begin{array}{ll}
1 & 1 \\
1 & 0
\end{array}\right)^{t}\right] \simeq \mathbb{Z}^{2} .
$$

Example 7. The Morse-Thue substitution: Let $\varphi$ denote the Morse-Thue substitution $(\varphi(1)=12, \varphi(2)=21)$.

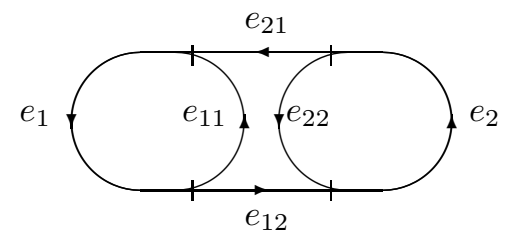

In this case, all transitions are allowed, and $K$ is as above. The subcomplex $S$ has a single connected component and $E R=S$, so $l=1=k$. It follows from Theorem 4 that

$$
\check{H}^{1}\left(\mathcal{T}_{\varphi}\right) \simeq \underline{\lim }\left(\begin{array}{ll}
1 & 1 \\
1 & 1
\end{array}\right) \oplus \mathbb{Z} \simeq \mathbb{Z}[1 / 2] \oplus \mathbb{Z}
$$

Example 8. Disconnected ER: Let $\varphi$ be the substitution: $\varphi(1)=12341, \varphi(2)=$ $12, \varphi(3)=3423, \varphi(4)=42$. The subcomplex $S$ has two connected components associated with the two sets of allowed transitions, $\{11,41,21,23,12,42\}$ and $\{34\}$, while $E R$ consists of two connected components associated with $\{21,11,23\}$ and $\{34\}$. Then $l=0, k=2$. According to Theorem $4, \breve{H}^{1}\left(\mathcal{I}_{\varphi}\right) \simeq \underset{\lim }{\longrightarrow} A^{t} / G$, where $G \simeq \mathbb{Z}$ and $A$ is the transition matrix for $\varphi$.

The details of the proof of Theorem 4 allow us to determine $\check{H}^{1}\left(\mathcal{T}_{\varphi}\right)$ more precisely. First, $\tilde{H}^{0}(S) \simeq \mathbb{Z}$ has basis $\left\{\hat{c}_{1}\right\}$, where $\hat{c_{1}}$ is the dual generator of a vertex $c_{1}$ in $e_{34}$. Then $\left\{\delta \hat{c}_{1}=\hat{e}_{3}-\hat{e}_{4}\right\}$ extends to the basis $\left\{\hat{e}_{3}-\hat{e}_{4}, \hat{e}_{1}, \hat{e}_{2}, \hat{e}_{3}\right\}$ of $H^{1}(K, S)$. Since $g_{1}^{*}$ has matrix $A^{t}$ with respect to the basis $\left\{\hat{e}_{1}, \hat{e}_{2}, \hat{e}_{3}, \hat{e}_{4},\right\}, g_{1}^{*}$ has matrix

$$
\left(\begin{array}{cccc}
1 & 0 & -1 & 0 \\
0 & 2 & 1 & 1 \\
0 & 1 & 1 & 0 \\
0 & 0 & 2 & 2
\end{array}\right)
$$

with respect to the basis $\left\{\hat{e}_{3}-\hat{e}_{4}, \hat{e}_{1}, \hat{e}_{2}, \hat{e}_{3}\right\}$. It follows that

$$
\check{H}^{1}\left(\mathcal{T}_{\varphi}\right) \simeq \lim _{\longrightarrow} A_{1} \oplus \mathbb{Z}^{l} \simeq \underline{\lim }\left(\begin{array}{lll}
2 & 1 & 1 \\
1 & 1 & 0 \\
0 & 2 & 2
\end{array}\right) .
$$




\section{REFERENCES}

[AP] J.E. Anderson and I.F. Putnam, Topological invariants for substitution tilings and their associated $C^{*}$-algebras, Ergodic Theory \& Dynamical Systems 18 (1998), 509-537. MR.1631708 (2000a:46112)

[BD] M. Barge and B. Diamond, A complete invariant for the topology of one-dimensional substitution tiling spaces, Ergodic Theory \& Dynamical Systems 21 (2001), 1333-1358. MR1855835 (2002k:37026)

[BJV] M. Barge, J. Jacklitch, and G. Vago, Homeomorphisms of one-dimensional inverse limits with applications to substitution tilings, unstable manifolds, and tent maps, Contemporary Mathematics 246, Amer. Math. Soc., Providence, RI (1999), 1-15. MR1732368 (2000j:37016)

[BJKR] O. Bratteli, P. Jorgenson, K. Kim, and F. Roush, Decidability of the isomorphism problem for stationary AF-algebras and the associated ordered simple dimension groups, Ergodic Theory \& Dynamical Systems 21 (2001), 1625-1655. MR.1869063 (2002h:46088)

[Dur] F. Durand, A characterization of substitutive sequences using return words, Discrete Math. 179 (1998), 89-101. MR1489074 (99g:68157)

[Mo] B. Mossé, Puissances de mots et reconnaissabilité des points fixes d'une substitution, Theoretical Computer Science 99 (1992), 327-334. MR.1168468 (93f:68076)

[So] B. Solomyak, Nonperiodicity implies unique composition for self-similar translationally finite tilings, Discrete Comput. Geometry 20 (1998), 265-279. MR1637896 (99f:52028)

Department of Mathematics, Montana State University, Bozeman, Montana 59717

E-mail address: barge@math.montana.edu

Department of Mathematics, College of Charleston, Charleston, South Carolina 29424

E-mail address: diamondb@cofc.edu 